\title{
Appropriate Treatment
}

National Cancer Institute

\section{Source}

National Cancer Institute. Appropriate Treatment. NCI Thesaurus. Code C101247.

Correct activity that produces an effect, or that is intended to alter the course of a disease in a patient or population. This is a general term that encompasses the medical, social, behavioral, and environmental acts that can have preventive, therapeutic, or palliative effects. (ACC) 